\title{
Pulmonary toxicities of molecular targeted antineoplastic agents: a single-center 10-year experience
}

\author{
Min-Young Lee ${ }^{1}$, Seug Yun Yoon ${ }^{1}$, Kyoung Ha Kim ${ }^{1}$ Namsu Lee ${ }^{1}$, Ha Youn Kim²,3 Jung Hwa Hwang ${ }^{2}$, \\ and Jong-Ho Won ${ }^{1}$
}

${ }^{1}$ Division of Hematology and Oncology, Department of Internal Medicine, ${ }^{2}$ Department of Radiology, Soonchunhyang University Seoul Hospital, Seoul; ${ }^{3}$ Department of Radiology, Daejeon Eulji Medical Center, Eulji University, Daejeon, Korea

Received: June 12, 2020

Revised : October 4, 2020

Accepted: October 22, 2020

\section{Correspondence to}

Jong-Ho Won, M.D.

Division of Hematology

and Oncology, Department

of Internal Medicine,

Soonchunhyang University

Seoul Hospital, 59 Daesagwan-ro,

Yongsan-gu, Seoul 04401, Korea

Tel: +82-2-709-9203

Fax: $+82-2-709-9200$

E-mail:jhwon@schmc.ac.kr https://orcid.org/0000-00016176-1442
Background/Aims: A better understanding of cancer cell biology has led to the discovery and development of several new targeted agents for cancer. These drugs are widely used in cancer treatment and have good toxicity profiles. However, some patients are extremely sensitive to these drugs and can develop severe toxicities. Among the toxicities, pulmonary complications are infrequent with most targeted therapies. This study aimed to identify the radiologic pulmonary complications in various targeted therapies and to analyze the characteristics of patients with pulmonary toxicity.

Methods: We retrospectively reviewed the medical records and chest image findings of 644 patients who were treated with targeted antineoplastic agents at Soonchunhyang University Hospital between May 2005 and September 2014.

Results: Of these 644 patients, 90 (14.0\%) developed pulmonary complications as noted on chest computed tomography. Among these patients, 15 (2.3\%) developed drug-related pulmonary toxicities. Treatment with targeted agents was discontinued in all patients, while 11 patients were simultaneously treated with glucocorticoids. Three patients died of drug-related pulmonary toxicity.

Conclusions: During targeted therapy, clinicians should assess for pulmonary toxicities and symptoms that occur with dyspnea. If drug-induced pulmonary toxicities are suspected, imaging studies should be performed immediately, and the possibility of variable radiological patterns should be considered. Discontinuing the use of implicated causative agents and treatment with glucocorticoids resulted in an improvement in both symptoms and imaging findings, but some patients still experienced fatal pulmonary toxicities.

Keywords: Molecular targeted therapy; Drug-related side effects and adverse reactions; Interstitial lung diseases; Lung injury

\section{INTRODUCTION}

Recent advances in cancer molecular biology have led to a new era of molecular-targeted therapy. Targeted antineoplastic agents, including monoclonal antibodies
(mAbs), tyrosine kinase inhibitors (TKIs), and mammalian target of rapamycin inhibitors, are commonly used in the treatment of various malignancies and are effective in cancer treatment [1]. However, use of these agents has caused unsuspected adverse drug reactions that are 
different from those caused by treatment with conventional systemic cytotoxic chemotherapy. In recent years, pulmonary toxicities associated with molecular targeted therapies have been reported. These case reports demonstrated that doctors should assess for pulmonary toxicities and perform proper management as these toxicities show significant variability and can cause fatal respiratory failure [2-8]. However, these reports were limited by the difficulty in distinguishing different diseases based on the radiologic findings, such as infection.

Therefore, this study aimed to retrospectively review the pulmonary toxicities caused by the use of molecular targeted agents and to analyze the various radiologic findings and clinical outcomes.

\section{METHODS}

\section{Study populations}

A total of 644 patients were treated with molecular targeted antineoplastic agents at Soonchunhyang University Seoul Hospital between May 2005 and September 2014. Targeted antineoplastic agents included mAbs (rituximab, cetuximab, bevacizumab, and trastuzumab) and TKIs (gefitinib, imatinib, erlotinib, and sorafenib). We searched for patients who underwent chest computed tomography (CT) or high-resolution CT (HRCT) scans during the course of targeted therapy. Of the 644 patients, 364 underwent chest CT or HRCT scans during the course of targeted therapy. Two radiologists (one chest radiologist with 15 years of experience in performing chest radiology and a junior resident in radiology) retrospectively reviewed the chest radiographs and CT scans of the 364 patients. After conducting a review of the radiological imaging, we identified 90 patients who presented with pulmonary complications on chest imaging. The medical records of these patients were reviewed by two clinical physicians in order to establish a diagnosis of drug-related pulmonary toxicity. The study was approved by the Institutional Review Board of Soonchunhyang University Hospital, Seoul, Korea (IRB No. 2013-01-008). Written informed consent by the patients was waived due to a retrospective nature of our study.

\section{Patterns of radiologic pulmonary complications}

Two CT scan devices (Sensation 16- and 64-section, Sie- mens Medical Solutions, Erlangen, Germany) were used in this study. The images were obtained volumetrically using multi-detector CT scan devices with o.6-mm or 1-mm collimation and 1-mm (high-spatial-frequency algorithm) or 3-mm (standard algorithm) reconstruction.

The CT findings were interpreted based on the recommendations of the nomenclature committee of the Fleischner Society $[9,10]$. Chest CT or HRCT images were assessed for major radiographic abnormalities: (1) air-space abnormalities, including air-space consolidation and ground-glass opacity; (2) interstitial abnormalities, including intralobular and interlobular septal thickening, bronchovascular bundle thickening, and honeycombing; (3) nodules and micronodules; (4) airway abnormalities, including bronchial wall thickening, bronchial dilatation, and air trapping; (5) cardiovascular abnormalities, including cardiomegaly, pulmonary thromboembolism (PTE), and pulmonary arterial enlargement; and (6) other findings, including mediastinal lymphadenopathy and pleural effusion. Anatomical distribution was classified as central, peripheral, peribronchovascular, or random. The zonal predominance of abnormalities was assessed as upper, middle, lower, or random. The distribution of parenchymal opacification was divided into the following patterns: diffuse, focal, or patchy patterns.

The semi-quantitative scoring system was used to evaluate the different abnormalities, including air-space abnormalities, interstitial abnormalities, nodules, and micronodules. Each abnormality was scored according to the involved area of the lung parenchyma: grade 1, $1 \%$ to $25 \%$; grade $2,26 \%$ to $50 \%$; grade $3,51 \%$ to $75 \%$; and grade $4,76 \%$ to $100 \%$ of the total lung. Airway abnormalities were scored using the method mentioned above. Cardiomegaly was classified as follows: mild, cardiothoracic (C-T) ratio of 0.5 to 0.6 , moderate, 0.6 to 0.7 ; and severe, $>0.7$. Pulmonary arterial enlargement was defined as an increase in the diameter of the main pulmonary artery (> $30 \mathrm{~mm}$ ) and right and left pulmonary arteries (>15 $\mathrm{mm}$ ). Lymphadenopathy was classified based on its short-axis diameter: mild, < $1 \mathrm{~cm}$; moderate, 1 to $1.5 \mathrm{~cm}$; and severe, $>1.6 \mathrm{~cm}$. Pleural effusion was defined as the distance between the inner costal margin and meniscus of the pleural fluid on axial CT scan: small, $<1.5 \mathrm{~cm}$; moderate, 1.5 to $4.5 \mathrm{~cm}$; and large, $>4.5 \mathrm{~cm}$. Evaluation of radiologic abnormalities and final diagnoses were made by the two 
observers.

Based on the CT analysis, targeted therapy-related pulmonary complications were categorized as follows: pneumonitis, nonspecific acute interstitial lung disease (ILD), organizing pneumonia, eosinophilic pneumonia, pulmonary fibrosis, bronchiolitis, pulmonary edema, adult respiratory distress syndrome (ARDS), diffuse alveolar hemorrhage (DAH), PTE, and pleural effusion. In cases of equivocal CT findings and lung parenchymal lesions with pleural or cardiovascular abnormalities, we selected one or more CT patterns. Each radiologic abnormality was defined as follows: (1) pneumonitis: interstitial abnormality with no remarkable lung fibrosis and less frequent air space abnormality, with CT patterns similar to that of idiopathic interstitial pneumonias, including non-specific interstitial pneumonia, desquamative interstitial pneumonia, and lymphoid interstitial pneumonia; (2) non-specific acute ILD: diffuse and less frequently patchy interstitial abnormality, which is mostly associated with ground-glass opacity and airspace consolidation and cannot be well categorized as the abovementioned pneumonitis pattern; (3) organizing pneumonia: similar CT features with cryptogenic organizing pneumonia of idiopathic form; (4) eosinophilic pneumonia: CT features reflecting Loeffler's disease or chronic eosinophilic pneumonia; (5) pulmonary fibrosis: irregular reticular opacities with traction bronchial/ bronchiectasis commonly accompanied by honeycombing and zonal predilection of peripheral, subpleural, and lower lung or diffuse involvement; (6) bronchiolitis: poorly defined centrilobular nodules or branching densities most easily identified in the lung periphery; (7) pulmonary edema: diffuse or patchy ground-glass opacities or consolidations and smooth interstitial thickening with dependent, perihilar, or lower lung predominance; (8) ARDS: diffuse or patchy ground-glass opacities or consolidations with geographic pattern and sometimes centrilobular or lobular opacities, and inconspicuous septal lines, which show dependent and basal predominance; and (9) DAH: patchy or diffuse ground-glass opacities or consolidations, frequently associated with poorly defined centrilobular nodules, and interlobular septal thickening developing over days after the acute episode. Preexisting lung diseases were also evaluated and included emphysema, nonspecific post-inflammatory sequelae, and known neoplasms.

\section{Patient eligibility and case definitions}

A diagnosis of drug-related pulmonary toxicity requires the presence of the following clinical conditions [11,12]: (1) new onset symptoms after administering targeted therapy; (2) lack of evidence of infectious cause; and (3) radiologic findings defined as pneumonitis or nonspecific acute interstitial disease by radiologists.

The exclusion criteria for this study were as follows: (1) evidence of infection based on the results of the investigation of the infectious cause and clinical symptoms or improvement after treatment with antibiotics; (2) history of ILD; (3) evidence of primary cancer progression; (4) history of discontinuation of previous targeted therapy due to the occurrence of another side effect; (5) suspected lung lesions detected on chest CT or HRCT before receiving targeted therapy; or (6) completion of the targeted therapy without clinical suspicion.

\section{Data collection}

The following demographic data, disease characteristics, and treatment details were reviewed: age, sex, Eastern Cooperative Oncology Group (ECOG) performance status at diagnosis, cancer diagnosis, type of targeted agent, treatment method, treatment duration, response, comorbidity, survival duration, and laboratory findings (complete blood count and differential counts, blood chemistry tests, and $\mathrm{O}_{2}$ saturation during blood gas analysis).

\section{RESULTS}

\section{Incidence of pulmonary complications and pulmo-} nary toxicity according to the targeted agents used From the database, 644 patients treated with the targeted agents from May 2005 to September 2014 were identified. Of the 644 patients, 364 underwent chest CT or HRCT during the course of targeted agent therapy. After conducting a review of the radiological imaging, we identified 90 patients who presented with pulmonary complications on chest CT. Over 39 patients $(6.1 \%)$ developed infectious pulmonary complications, while 51 (7.9\%) had noninfectious pulmonary complications. A total of 15 patients (2.3\%) were diagnosed with drug-induced pulmonary toxicity during the course of targeted 


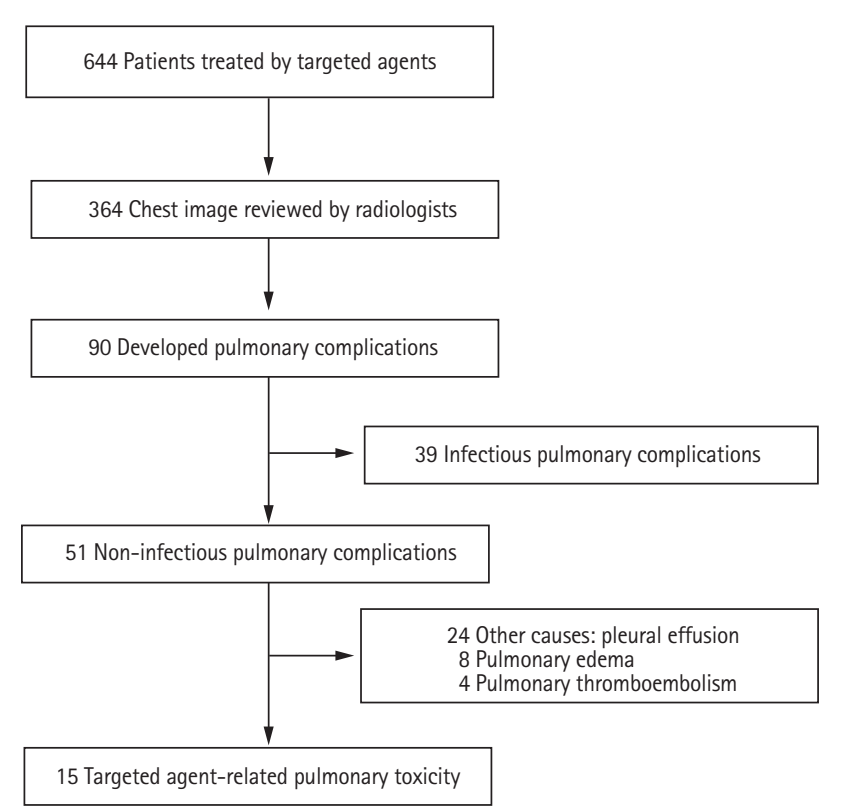

Figure 1. Flow chart of the patient selection process. Of 644 patients, 15 were diagnosed with drug-induced pulmonary toxicity during the course of targeted agent therapy.

therapy (Fig. 1). The radiological pulmonary complication rates were similar between $\mathrm{mAbs}(13.6 \%)$ and TKIs (14.3\%); meanwhile, TKIs (3.5\%) showed higher targeted agent-related pulmonary toxicity rates than $\mathrm{mAbs}(1.3 \%)$, but the difference was not statistically significant $(p=$ o.079) (Table 1). Treatment with gefitinib was most commonly associated with pulmonary toxicities (5.1\%), followed by use of cetuximab (3.6\%), erlotinib (3.4\%), imatinib (3.3\%), rituximab (2.9\%), and bevacizumab (0.7\%).

The clinical characteristics of these 15 patients are listed in Table 2. The patients' median age was 68 years (range, 45 to 83 ), and $53.3 \%$ of the patients were women. Twelve patients (80.0\%) had an ECOG performance status of o-1 and solid tumors; of these 12 patients, 10 were treated with a targeted agent as a palliative therapy. Three patients (20.0\%) had hematologic malignancy.

\section{Radiologic findings}

The CT findings of patients with noninfectious pulmonary toxicities related to the use of targeted agents are summarized in Table 3. The predominant CT findings were ground-glass opacities (70.6\%) and air-space consolidations (39.2\%). Moreover, interlobular septal thickening (58.8\%) and intralobular lines (41.2\%) were frequently observed; bronchovascular bundle thicken-

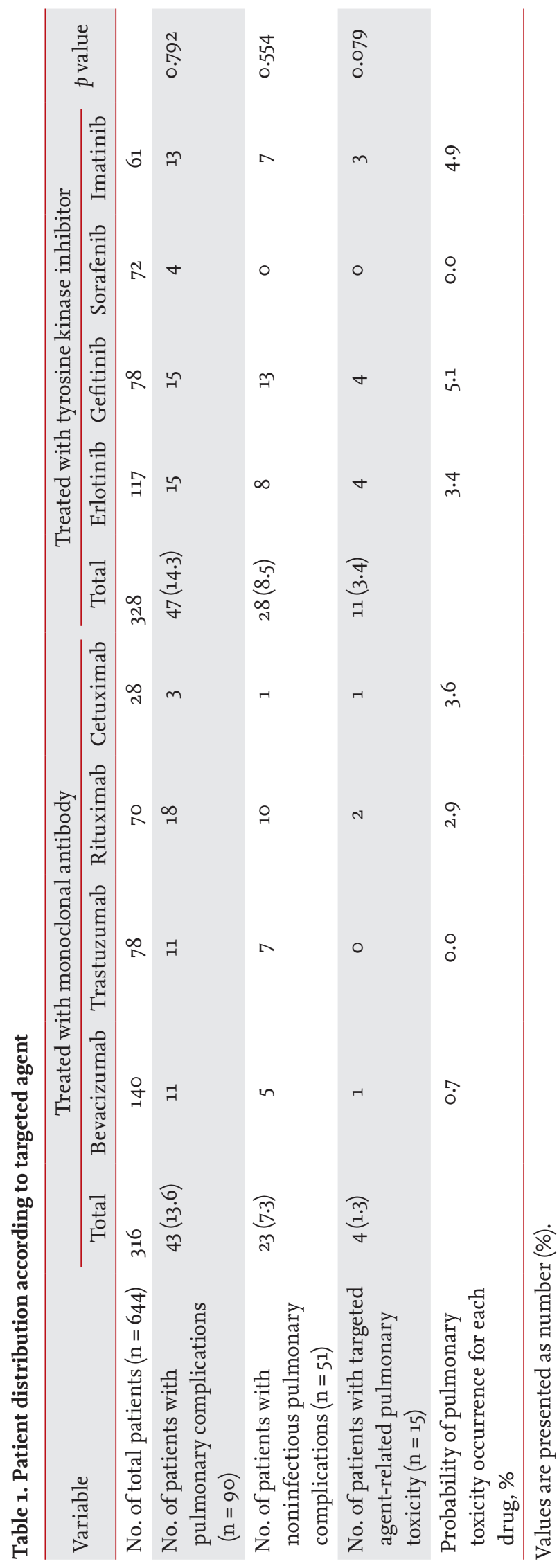


Table 2. Characteristics of patients with targeted agent-related pulmonary toxicity $(n=15)$

\begin{tabular}{|c|c|}
\hline Characteristic & Value \\
\hline Time to pulmonary toxicity, mo & $2.3(0.1-8.5)$ \\
\hline Age, yr & $68(45-83)$ \\
\hline \multicolumn{2}{|l|}{ Sex } \\
\hline Male & $7(46.7)$ \\
\hline Female & $8(53 \cdot 3)$ \\
\hline \multicolumn{2}{|l|}{ ECOG } \\
\hline o & $2(13 \cdot 3)$ \\
\hline 1 & $10(66.7)$ \\
\hline 2 & $3(20.0)$ \\
\hline \multicolumn{2}{|l|}{3,4} \\
\hline \multicolumn{2}{|l|}{ Type of cancer } \\
\hline Solid tumor & $12(80.0)$ \\
\hline Lung cancer & $5(33 \cdot 3)$ \\
\hline Pancreatic cancer & $3(20.0)$ \\
\hline GIST & $2(13 \cdot 3)$ \\
\hline Stomach cancer & $1(6.7)$ \\
\hline Rectal cancer & $1(6.7)$ \\
\hline Hematologic cancer & $3(20.0)$ \\
\hline DLBCL & $2(13 \cdot 3)$ \\
\hline CML & $1(6.7)$ \\
\hline \multicolumn{2}{|l|}{ Treatment setting of solid tumors } \\
\hline Adjuvant & $2(16.7)$ \\
\hline Palliative & $10(83.3)$ \\
\hline
\end{tabular}

Values are presented as median (range) or number (\%). ECOG, Eastern Cooperative Oncology Group; GIST, gastrointestinal stromal tumor; DLBCL, diffuse large B-cell lymphoma; CML, chronic myeloid leukemia.

ing (19.6\%) was also noted. None of the study patients experienced honeycombing. Nodules (2.0\%) and micronodules (9.8\%) were not frequently observed. Mild bronchial wall thickening (5.9\%) and bronchial dilatation $(1.9 \%)$ were only noted in a small number of patients. On cardiovascular evaluation, mild cardiomegaly (21.6\%), PTE (7.8\%), and mild pulmonary artery enlargement $(7.8 \%)$ were detected. Other findings included mild mediastinal lymphadenopathy (21.6\%) and pleural effusion (54.9\%). The common anatomic distributions were peripheral (39.2\%) and lower lung zonal (39.2\%) predominance. The diffuse pattern (47.1\%) was the most commonly observed distribution of parenchymal opaci- fication (Table 3). The radiologic patterns of pulmonary toxicities in our study and the related targeted chemotherapeutic agents are described in Table 4.

\section{Symptoms and time span of pulmonary toxicity}

All patients who developed targeted agent-related pulmonary toxicities had symptoms. Dyspnea was the most common initial symptom and was prevalent in 12 of 15 (80.0\%) symptomatic patients. Two patients had a cough and one had pleuritic pain. The median duration of pulmonary toxicity occurrence from treatment of targeted agents was 70 days (range, 3 to 203).

\section{Risk factors of pulmonary toxicity}

Older age and lower serum albumin level were the common risk factors in patients with targeted agent-related pulmonary toxicities (Table 5). In the multivariate analysis, only older age was significantly associated with the development of pulmonary toxicity after adjusting for other variables (odds ratio, 1.052; 95\% confidence interval, 1.004 to 1.103) (Table 6). The fitness of this model was confirmed using the Hosmer-Lemeshow goodness of fitness test $(p=0.253)$.

\section{Treatment of pulmonary toxicities and responses}

As targeted agent-induced pulmonary toxicity was suspected, targeted therapy was discontinued in all patients. After discontinuing this therapy, 11 patients (73.3\%) were treated with systemic glucocorticoids. Among these patients, five (45.5\%) was treated with methylprednisolone as a steroid pulse therapy at a dose of 250 to $500 \mathrm{mg} /$ day for 3 days. Six patients (54.5\%) received prednisolone at a dose of 0.5 to $1.0 \mathrm{mg} / \mathrm{kg} /$ day. The initial dose was 0.87 $\mathrm{mg} / \mathrm{kg} /$ day and was gradually tapered from 4 to 12 weeks. The pulmonary toxicity in eight patients eventually resolved. The patients' radiologic findings improved within a mean duration of 49 days (range, 5 to 365) after the occurrence of pulmonary toxicity. Among patients with symptomatic and significant radiologic findings who completely recovered, three were re-treated with a lower dose of the equal drug in combination with glucocorticoids. In addition, these patients did not experience recurrence of pulmonary toxicity.

Four patients did not show resolution of pulmonary toxicity in the chest images. The radiologic imaging of one patient showed persistent pulmonary toxicity, but 
Table 3. Radiologic findings of patients with noninfectious pulmonary complications $(\mathbf{n}=\mathbf{5 1})$

\begin{tabular}{|c|c|c|c|}
\hline Variable & No. (\%) & CT grade & Number \\
\hline \multicolumn{4}{|l|}{ CT findings } \\
\hline \multicolumn{4}{|l|}{ Air-space abnormalities } \\
\hline \multirow[t]{4}{*}{ Air-space consolidations } & $20(39.2)$ & $\mathrm{I}$ & 14 \\
\hline & & II & 4 \\
\hline & & III & 1 \\
\hline & & IV & 1 \\
\hline \multirow[t]{4}{*}{ Ground-glass opacities } & $36(70.6)$ & $\mathrm{I}$ & 16 \\
\hline & & II & 6 \\
\hline & & III & 3 \\
\hline & & IV & 11 \\
\hline \multicolumn{4}{|l|}{ Interstitial abnormalities } \\
\hline \multirow[t]{4}{*}{ Intralobular lines } & $21(41.2)$ & $\mathrm{I}$ & 10 \\
\hline & & II & 7 \\
\hline & & III & 3 \\
\hline & & IV & 1 \\
\hline \multirow[t]{4}{*}{ Interlobular septal thickenings } & $30(58.8)$ & I & 20 \\
\hline & & II & 7 \\
\hline & & III & 2 \\
\hline & & IV & 1 \\
\hline \multirow[t]{2}{*}{ Bronchovascular bundle thickenings } & $10(19.6)$ & $\mathrm{I}$ & 8 \\
\hline & & II & 2 \\
\hline Honeycombing & o & & \\
\hline \multicolumn{4}{|l|}{ Nodules and micronodules } \\
\hline Nodules & $1(2.0)$ & I & 1 \\
\hline Micronodules & $5(9.8)$ & I & 5 \\
\hline \multicolumn{4}{|l|}{ Airway abnormalities } \\
\hline Bronchial wall thickenings & $3(5 \cdot 9)$ & I & 3 \\
\hline Bronchial dilatations & $1(1.9)$ & $\mathrm{I}$ & 1 \\
\hline Air trappings & $1(1.9)$ & & \\
\hline \multicolumn{4}{|l|}{ Cardiovascular abnormalities } \\
\hline Cardiomegaly & $11(21.6)$ & Mild & 11 \\
\hline Pulmonary artery enlargement & $4(7.8)$ & Mild & 4 \\
\hline Pulmonary thromboembolism & $4(7.8)$ & & \\
\hline \multicolumn{4}{|l|}{ Others } \\
\hline Lymphadenopathy & $11(21.6)$ & Mild & 11 \\
\hline \multirow{2}{*}{ Pleural effusions } & $28(54.9)$ & Small & 26 \\
\hline & & Moderate & 2 \\
\hline \multicolumn{4}{|l|}{ Anatomic distribution } \\
\hline Central & $2(3.9)$ & & \\
\hline Peripheral & $20(39.2)$ & & \\
\hline Peribronchovascular & $8(15 \cdot 7)$ & & \\
\hline Random & $16(31.4)$ & & \\
\hline \multicolumn{4}{|l|}{ Zonal predominance } \\
\hline Upper & $7(13.7)$ & & \\
\hline Middle & $4(7.8)$ & & \\
\hline Lower & $20(39.2)$ & & \\
\hline Random & $15(29.4)$ & & \\
\hline \multicolumn{4}{|l|}{ Distribution of parenchymal opacification } \\
\hline Diffuse & $24(47 \cdot 1)$ & & \\
\hline Patchy & $13(25 \cdot 5)$ & & \\
\hline Focal & o & & \\
\hline
\end{tabular}

CT, computed tomography. 
Table 4. Pulmonary complications and associated targeted agents

\begin{tabular}{lll}
\hline Radiologic finding & \multicolumn{1}{c}{ Molecular antibodies } & \multicolumn{1}{c}{ Tyrosine kinase inhibitors } \\
\hline Pneumonitis & Rituximab and cetuximab & Gefitinib and imatinib \\
NAILD & Bevacizumab and rituximab & Erlotinib, gefitinib, and imatinib \\
Organizing pneumonia & Trastuzumab and cetuximab & Erlotinib and imatinib \\
Eosinophilic pneumonia & Rituximab & Gefitinib and imatinib \\
Pulmonary fibrosis & None & Erlotinib, gefitinib, and imatinib \\
Bronchiolitis & None & None \\
Pulmonary edema & Rituximab and cetuximab & Gefitinib \\
ARDS & Rituximab & Erlotinib and gefitinib \\
Diffuse alveolar hemorrhage & Rituximab & Erlotinib and gefitinib \\
Pulmonary thromboembolism & Bevacizumab & Gefitinib \\
Pleural effusion & Bevacizumab and trastuzumab & Erlotinib and gefitinib \\
\hline
\end{tabular}

NAILD, nonspecific acute interstitial disease; ARDS, adult respiratory distress syndrome.

Table 5. Univariate analysis of risk factors for targeted agent-related pulmonary toxicity

\begin{tabular}{|c|c|c|c|}
\hline \multirow{2}{*}{ Variable } & \multicolumn{2}{|c|}{ Targeted agent-related pulmonary toxicity } & \multirow{2}{*}{$p$ value } \\
\hline & No $(n=629)$ & Yes $(n=15)$ & \\
\hline Age, yr & $58.05 \pm 13.73$ & $65.93 \pm 10.25$ & $0.028^{\mathrm{a}}$ \\
\hline Male sex & $324(51.5)$ & $7(46.7)$ & 0.711 \\
\hline \multicolumn{4}{|l|}{ Laboratory findings } \\
\hline Leukocytes, $\times 10^{3} / \mu \mathrm{L}$ & $7.93 \pm 14.49$ & $6.69 \pm 3.14$ & 0.742 \\
\hline Hemoglobin, g/dL & $11.15 \pm 1.97$ & $10.37 \pm 1.39$ & $0.129^{\mathrm{a}}$ \\
\hline Platelet, $\times 10^{3} / \mu \mathrm{L}$ & $248.82 \pm 136.48$ & $242.60 \pm 122.64$ & 0.861 \\
\hline Creatinine, mg/dL & $0.90 \pm 0.88$ & $0.77 \pm 0.22$ & 0.583 \\
\hline AST, IU/L & $36.37 \pm 43.42$ & $24.67 \pm 6.98$ & 0.297 \\
\hline ALT, IU/L & $26.52 \pm 29.3$ & $20.33 \pm 8.72$ & 0.415 \\
\hline Albumin, g/dL & $3.68 \pm 0.62$ & $3.37 \pm 0.40$ & $0.049^{\mathrm{a}}$ \\
\hline
\end{tabular}

Values are presented as mean \pm standard deviation or number (\%).

AST, aspartate transaminase; ALT, alanine transaminase.

${ }^{a}$ Data used for multivariate analysis.

the patient did not develop severe symptoms. Another three patients $(20.0 \%)$ died due to target agent-related pulmonary toxicity. The median duration of death after pulmonary toxicity was 0.2 months. Two patients were treated with erlotinib for pancreatic cancer, while one was treated with cetuximab for rectal cancer. The other seven patients died due to disease progression, one died of cardiac arrest due to massive upper gastrointestinal bleeding, and one died of respiratory arrest due to aspiration.

\section{DISCUSSION}

Cancer treatments have significantly changed with the improved understanding of cancer cell biology, which has led to the development of novel molecular targeted antineoplastic agents. As use of targeted agents becomes more frequent for cancer treatment, physicians must assess for possible toxicities. Since the first report of gefitinib-associated ILD was published in Japan [13], pulmonary toxicities associated with the use of molecular targeted agents have rarely been reported. The exact in- 
Table 6. Multivariate analysis of risk factors for targeted agent-related pulmonary toxicity

\begin{tabular}{|c|c|c|c|c|}
\hline \multirow{2}{*}{ Variable } & \multirow{2}{*}{ Significance ( $p$ value) } & \multirow{2}{*}{ Adjusted odds ratio } & \multicolumn{2}{|c|}{$95 \% \mathrm{CI}$} \\
\hline & & & Lower & Upper \\
\hline Mean age, $\mathrm{yr}^{\mathrm{a}}$ & 0.034 & 1.052 & 1.004 & 1.103 \\
\hline Hemoglobin, g/dL ${ }^{b}$ & 0.368 & 0.866 & 0.632 & 1.185 \\
\hline Albumin, $\mathrm{g} / \mathrm{dL}^{\mathrm{c}}$ & 0.404 & 0.668 & 0.259 & 1.722 \\
\hline
\end{tabular}

CI, confidential interval.

$\mathrm{a}(\mathrm{x}+1$ years vs. $\mathrm{x}$ years $)$.

b,c $(x+1$ g/dL vs. $x \mathrm{~g} / \mathrm{dL})$.

cidence of targeted agent-induced pulmonary toxicities remains unclear, with many studies reporting a variable incidence of these adverse events, from $0.2 \%$ to $10.9 \%$ [14]. In our study, the incidence of targeted agent-induced pulmonary toxicities was $2.3 \%$, which is consistent with the findings from previous studies $[14,15]$. Our study included both mAbs and TKIs, and pulmonary complications occurred similarly in both groups. The drug-related pulmonary toxicity was found to be higher in TKIs than in mAbs, but no significant difference was observed.

Since a variety of pulmonary complications can be observed on chest CT images in patients treated with targeted therapy, drug-induced pulmonary toxicity should be diagnosed based on the clinical symptoms and imaging findings. Many patients develop symptoms, including dyspnea, cough, and fever, which are mostly confined to the lungs [16]. The patterns and severity of clinical manifestations may differ depending on the patient's underlying illness and the relevant drug [15]. As previously reported in other studies, the most common symptom was dyspnea. This result suggests that physicians should be concerned about patients with newly developed respiratory symptoms during the course of molecular-targeted agent treatment.

A diagnosis of pulmonary toxicity should be considered in patients with a history of exposure to molecular targeted agents, who present with new or progressive respiratory symptoms [17]. Most importantly, the diagnosis is made after excluding other possible causes, including infections, iatrogenic intra-alveolar hemorrhage, radiotherapy-induced adverse effects, transfusion reactions, and progression of the underlying neoplasm. This is because the prognostic and therapeutic implications of these factors are extremely different $[16,18]$.
Physicians should perform immediate imaging studies (chest radiographs and CT scan), appropriate cultures, serologic tests, and bronchoscopy with bronchoalveolar lavage in patients who may be at risk [14]. Although chest radiography is commonly performed in patients with respiratory manifestations as an initial diagnostic imaging modality, chest CT is more sensitive in detecting parenchymal lung diseases. Therefore, the recognition of various CT manifestations and patterns of targeted agent-induced pulmonary toxicities is essential for timely diagnosis and appropriate management of any adverse events.

Risk factors for drug-induced pulmonary toxicity have been identified in several retrospective studies. Many variable risk factors such as preexisting pulmonary fibrosis, poor performance status, previous thoracic irradiation, smoking, older age, male sex, or hypoalbuminemia have been suggested $[1,11,12,19,20]$. In this study, older age was the only risk factor for drug-induced pulmonary toxicity. This is probably because the present study targeted a variety of drugs and diseases.

Pulmonary toxicity may respond to appropriate treatment; however, mortality has also been reported [16]. Although no randomized controlled trials have evaluated the treatment benefit, immediate discontinuation of the targeted agent and administration of systemic glucocorticoids with supportive care resulted in an improvement in both symptoms and radiographic findings [1,14,15]. The dose and duration of glucocorticoids varied in the literature. According to the severity of respiratory failure, high-dose methylprednisolone (500 $\mathrm{mg}$ to 1,000 $\mathrm{mg} /$ day for 3 days) was recommended [7]. In this study, the administration of targeted agents was discontinued in all patients with or without treatment of systemic glucocorticoids; among them, 53.3\% patients showed reso- 
lution of symptoms and improvement in radiological findings. Therefore, careful administration of systemic glucocorticoids, in accordance with the patient's baseline comorbidities and other possible infectious conditions, is necessary. In addition, the beneficial impact of systemic glucocorticoids, according to relevant targeted agents, needs to be validated in future studies.

In our study, among the patients who discontinued taking the targeted drugs because of pulmonary toxicity, three were re-treated with equivalent drugs after resolution of clinical symptoms and radiological manifestations. Almost all patients who were treated with the same drug in combination with low-dose glucocorticoid did not experience a recurrence of pulmonary toxicity. However, a few studies have reported the re-administration of an equal molecular agent. Peerzada et al. [14] reported that four (36.4\%) among 11 patients had relapsed after re-treatment with imatinib, following the diagnosis of imatinib-induced pulmonary toxicity. Thus, physicians must carefully determine whether chemotherapy should be discontinued or not in patients receiving a molecular agent as the main therapy for cancer, and further studies are needed.

Previous clinical trial reports and the study summaries of these targeted agents have reported various life-threatening respiratory side effects. Approximately one-third of reported cases are fatal [1]. Therefore, oncologists must assess for signs of toxicities early to prevent mortality. Targeted agent-induced pulmonary toxicity can be difficult to diagnose due to the variations in patients' radiological findings. Therefore, the causative drug must be recognized, together with an understanding of the radiologic manifestations of pulmonary toxicity caused by these targeted agents, in order to provide the appropriate management [17]. Oncologists, in conjunction with radiologists, should be knowledgeable of the clinical manifestations and variable radiologic manifestations of drug-induced pulmonary toxicity, which can increase in incidence with the treatment of diverse types of molecular targeted agents.

Drugs have been implicated in lung injury as a result of direct pharmacological action, persistence, or metabolism in the tissue, or via the production of a reactive metabolite. There is limited knowledge on how the timing of drug administration or drug interactions may interfere with the repair mechanisms or modulate the expression of pulmonary toxicity. Unlike conventional chemotherapy, where the actions are systemic and direct as results of biotransformation or cell injury, the use of targeted antineoplastic agents more likely affects the epithelia via a pharmacological effect. Some studies have reported the possibility that low albumin levels are a risk factor for mAbs or TKIs, especially gefitinib because a low albumin level is one of the markers of malnutrition [11,12]. Hence, future studies should identify other risk factors for the development of adverse pulmonary reactions and biological markers that can be used to predict the occurrence of pulmonary toxicity. Proteomics and genomics will hopefully identify the candidates for this role [21].

Several limitations should be considered when interpreting our findings: the retrospective design of the study, the small number of patients, the heterogeneity of molecular targeted agents, and baseline diseases. In the future, a prospective study with a large number of patients treated with independent agents should be conducted.

In conclusion, the results from this study suggest that pulmonary toxicities associated with the use of molecular targeted agent therapy rarely occur but can be potentially fatal. Hence, physicians need to be aware of the varied clinical and radiological manifestations to provide appropriate management.

\section{KEY MESSAGE}

1. Various radiologic pulmonary complications can be observed in patients treated with targeted therapy.

2. Because drug-induced pulmonary toxicity can be fatal and requires immediate treatment, physicians should make appropriate treatment decisions by considering the changes in the patients' imaging findings and clinical symptoms.

\section{Conflict of interest}

No potential conflict of interest relevant to this article was reported. 


\section{Acknowledgments}

This work was supported in part by the Soonchunhyang University Research Fund.

\section{REFERENCES}

1. Barber NA, Ganti AK. Pulmonary toxicities from targeted therapies: a review. Target Oncol 2011;6:235-243.

2. Aparicio G, Calvo MB, Medina V, et al. Comprehensive lung injury pathology induced by mTOR inhibitors. Clin Transl Oncol 2009;11:499-510.

3. Arora A, Scholar EM. Role of tyrosine kinase inhibitors in cancer therapy. J Pharmacol Exp Ther 2005;315:971-979.

4. Burton C, Kaczmarski R, Jan-Mohamed R. Interstitial pneumonitis related to rituximab therapy. N Engl J Med 2003;348:2690-2691.

5. Kang W, Kim JS, Cho SH, Kim SK, Chang J, Park MS. Nonspecific interstitial pneumonitis after bortezomib and thalidomide treatment in a multiple myeloma patient. Yonsei Med J 2010;51:448-450.

6. Makris D, Scherpereel A, Copin MC, et al. Fatal interstitial lung disease associated with oral erlotinib therapy for lung cancer. BMC Cancer 2007;7:150.

7. Vahid B, Marik PE. Pulmonary complications of novel antineoplastic agents for solid tumors. Chest 2008;133:528538.

8. Vulsteke C, Dierickx D, Verbeken E, Wolter P, Thomas J, Schoffski P. Rituximab-induced fatal interstitial pneumonitis: case report. Leuk Lymphoma 2010;51:546-548.

9. Austin JH, Muller NL, Friedman PJ, et al. Glossary of terms for CT of the lungs: recommendations of the Nomenclature Committee of the Fleischner Society. Radiology 1996;200:327-331.

10. Hansell DM, Bankier AA, MacMahon H, McLoud TC, Muller NL, Remy J. Fleischner Society: glossary of terms for thoracic imaging. Radiology 2008;246:697-722.

11. Kang HJ, Park JS, Kim DW, et al. Adverse pulmonary reactions associated with the use of monoclonal antibodies in cancer patients. Respir Med 2012;106:443-450.

12. Beom SH, Kim DW, Sim SH, et al. Gefitinib-induced interstitial lung disease in Korean lung cancer patients. Cancer Res Treat 2016;48:88-97.

13. Inoue A, Saijo Y, Maemondo M, et al. Severe acute interstitial pneumonia and gefitinib. Lancet 2003;361:137-139.

14. Peerzada MM, Spiro TP, Daw HA. Pulmonary toxicities of tyrosine kinase inhibitors. Clin Adv Hematol Oncol 2011;9:824-836.

15. Min JH, Lee HY, Lim H, et al. Drug-induced interstitial lung disease in tyrosine kinase inhibitor therapy for nonsmall cell lung cancer: a review on current insight. Cancer Chemother Pharmacol 2011;68:1099-1109.

16. Dimopoulou I, Bamias A, Lyberopoulos P, Dimopoulos MA. Pulmonary toxicity from novel antineoplastic agents. Ann Oncol 2006;17:372-379.

17. Rossi SE, Erasmus JJ, McAdams HP, Sporn TA, Goodman PC. Pulmonary drug toxicity: radiologic and pathologic manifestations. Radiographics 2000;20:1245-1259.

18. Domingo C, Roig J. Neglected respiratory toxicity caused by cancer therapy. Open Respir Med J 2007;1:1-6.

19. Takano T, Ohe Y, Kusumoto M, et al. Risk factors for interstitial lung disease and predictive factors for tumor response in patients with advanced non-small cell lung cancer treated with gefitinib. Lung Cancer 2004;45:93-104.

20. Ando M, Okamoto I, Yamamoto N, et al. Predictive factors for interstitial lung disease, antitumor response, and survival in non-small-cell lung cancer patients treated with gefitinib. J Clin Oncol 2006;24:2549-2556.

21. Higenbottam T, Kuwano K, Nemery B, Fujita Y. Understanding the mechanisms of drug-associated interstitial lung disease. Br J Cancer 2004;91 Suppl 2:S31-S37. 